\title{
Laser Interaction with Material - Theory, Experiments and Discrepancies
}

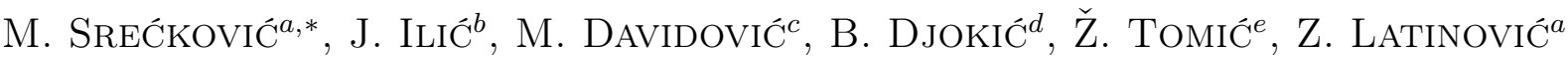 \\ AND D. DRUŽIJANIĆ ${ }^{a}$ \\ ${ }^{a}$ Faculty of Electrical Engineering, Belgrade, Serbia \\ ${ }^{b}$ Faculty of Mechanical Engineering, Belgrade, Serbia \\ ${ }^{c}$ Faculty of Civil Engineering, University of Belgrade, Belgrade, Serbia \\ ${ }^{d}$ Educon, Nova Varoš, Serbia \\ ${ }^{e}$ IRITEL AD, Belgrade, Serbia
}

\begin{abstract}
The experimental treatment of chosen material with laser beams, starting from continuous wave up to fs pulses, produces the necessity to find the common and sophisticated theoretical approaches to interaction modeling. For chosen materials, some laser treatment and damage analyses are performed. The provoked stresses and parameters of transport processes (penetration depth) are calculated by using the programs for electrical circuit analyses. Some inconsistencies in the treatment of large area laser-material interaction are discussed.
\end{abstract}

PACS numbers: 01.55.+b, 42.62.-b, 42.25.Bs, 81.40.Wx, 61.80.Ba

\section{Introduction}

The development of new laser types, ranging from continuous wave $(\mathrm{CW})$ lasers to atto-second pulse lasers (that used to be considered as pulses beyond the theoretical frontier), obliges the numerous approaches to interaction of laser beams with a material to be constantly updated. Apart from the models developed directly for the particular ranges of pulse durations, various laser dynamics, power densities and materials required new approaches. The possibility that, for the highest power densities regardless on the treated material, lasers produce explosive processes and total destruction should not be discarded. At lower power densities, of greater importance are many other factors not expressed by the chief optical and thermodynamic properties of treated materials (such as thermal conductivity, density, specific heat, coefficients of reflection, absorption and diffusion).

The detailed analyses of laser-matter interaction require the application of numerical methods. A specific approach is the modeling of general statistics where the fluctuations of beam parameters (polarization, power, pulse energy) are expressed by appropriate mathematical functions.

There were attempts to explain the discrepancies between the experiments and the laser induced damage threshold (LIDT) theory by the material (and its im-

* corresponding author; e-mail: esreckov@etf.rs purities) previous record. That led to the parallel development of models based on damage threshold, dielectric breakdown, Brillouin threshold, self-focusing, thermal lenses, thermal breakdown, etc. The field of pulse compression concerning refraction indices and its decrements is important for nonlinear electro-optic, acousto-optic and electromagnetic processes, and it is developed separately. Let us note that the photoelastic and electro-optic properties of crystals are strongly connected. Besides that, hydrodynamics-based models with plasma expansion and degeneracy of the electron gas for ultrashort laser pulses are developed [1-5].

The tendencies to explain the inconsistencies between the theory and experiments by general approaches involving the statistics of the phenomenon appeared. They include, in the same model, both pure materials and materials with impurities (bubbles, particles, dust). The state of surface roughness together with the specific shape of surface defects (scratches) is included by changing the approximation of internal field ( $\left.E_{\text {threshold }}\right)[2,6]$ and can be corrected by using the function of the refractive index. On the other hand, the right choice of the statistical function describing cumulative and single pulse exposition for experimental data fitting could be a problem. Thus, using the statistics of binomial and Poisson type seems to give good explanation for the causes of the inconsistencies [6].

For chosen materials, including pure metals, organic compounds, bio-materials, alloys, thin films and bulk materials, various laser exposures are performed. The dam- 
ages are analyzed by the methods of light microscopy, scanning electron microscopy (SEM), IR spectra, micro-hardness tests, energy distribution of X-ray (EDX), etc. For specific cases, the numerical estimations of both the temperature distributions and provoked stresses are calculated. By the implementation of the programs typically used in electric circuit analysis, the temperature distributions caused by laser-matter interaction are calculated. Many programs and references for the analysis of the damages provoked on the optical components and accessories along the pulse propagation direction are available.

Expected dependences that should be generally taken into account, thermal and other equations involving linear and nonlinear components of used parameters, as well as the estimations of the nonlinear constants of the materials are very complex in the case of modern materials. Besides rigorous theoretical calculations and complex equipment for measurements, we also studied the practical formulae which include laser intensity limiting as well as the evaluation of damage sizes, like crater diameter and depth. As an example, nonlinear part of the refractive index, $n_{2}$, can be found by knowing the dispersion parameter $V$ (Abbe value) and refractive index for Na line $n_{\mathrm{D}}$ (yellow) [7]. The attention has to be paid to the new categories of the discrepancies between the theory and the results of laser damages as well as to the expelled material at the damage place. Some problems include the interpretation of the relation between the pulse regime and damages (single or cumulative effects). Apart from standard electrical and optical techniques, such as microscopy, micro-hardness tests, etc., the results can also be evaluated by detailed image processing. In this way, the beam energy distribution and its multimode structure can be confirmed [8]. The model of mechanical stresses induced by laser beam propagation is evaluated by appropriate differential equations and by newly developed approaches as in $[9,10]$.

\section{Some experimental results}

In a way, the comparison of the shapes of fs- and ns-damages could be treated similarly as the comparison of the shapes of ns and free-generation regime damages. We treated transparent materials (glasses, composites), ceramics and semiconductors with pulsed beams from Q-switch $\mathrm{Nd}^{3+}$ : YAG (ns, $1060 \mathrm{~nm}$ ) and Ti:sapphire (fs, I and III harmonics) lasers. Some experimental results of ns and fs laser expositions are presented in Fig. 1. Depending on the chosen experimental conditions, various results have been obtained.

\section{Numerical results for material stresses provoked by laser beam}

The analysis of stresses provoked by laser beam propagating through material is linked to the theories based on the mechanics and physics of fractures and to the transport equations. During the analysis, thermal, Brillouin,
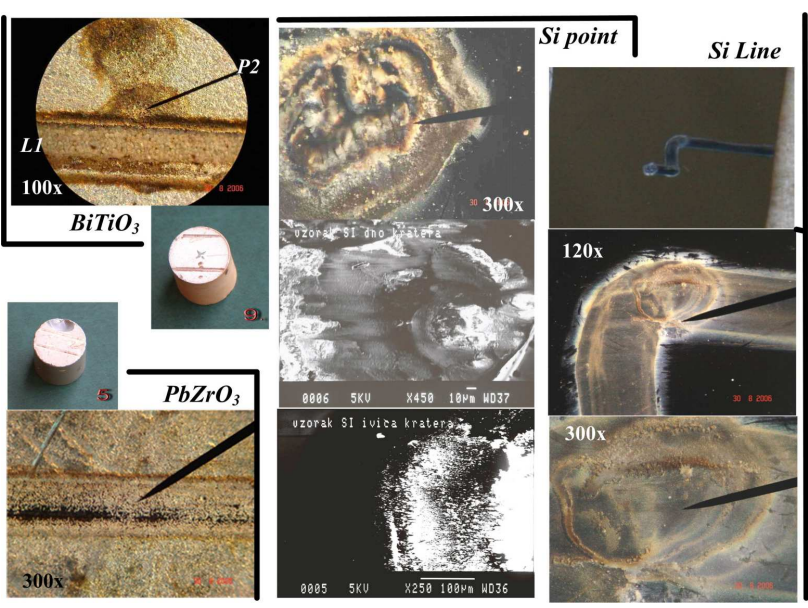

Fig. 1. Light microscopy (LM) and SEM micrographs of ceramics and semiconductor samples damaged by fs and ns pulses. Sample "BiTiO $3 " / " 9 " ~\left(\mathrm{BiTiO}_{3} ; \mathrm{LM}\right.$ $100 \times)$ : "P2", a crater by $\mathrm{Nd}^{3+}$ :YAG $(8 \mathrm{~ns}, 6 \mathrm{~mJ}, 20$ pulses); " $L 1$ ", a line by Spectra Physics' Spitfire Pro Ti:sapphire $(0.95 \mathrm{~W}$ average, I harmonic $-800 \mathrm{~nm}$; pulse duration - $40 \mathrm{fs}$, repetition rate $1 \mathrm{kHz}$, exposed $5 \mathrm{~min}$; displaced with small velocity). Sample "PbZrO $3 " / " 5 "\left(\mathrm{PbZrO}_{3} ; \mathrm{LM} 300 \times\right)$ : a line by fs beam (I harmonic - $800 \mathrm{~nm}$; exposed $2 \mathrm{~min}$; displaced with small velocity). Sample "Si point" (Si, 99\% purity; LM $300 \times$ and SEM $450 \times$ - crater bottom; SEM 250× - crater edge): a damage by Spectra Physics' Spitfire Pro Ti:sapphire (III harmonic $-266 \mathrm{~nm}$, exposed 25 min). Sample "Si line" (Si 99\% purity, LM 120×, LM $300 \times$ ): a line by fs beam (I harmonic - $800 \mathrm{~nm}$; exposed $1.5 \mathrm{~min}$; displaced with small velocity).

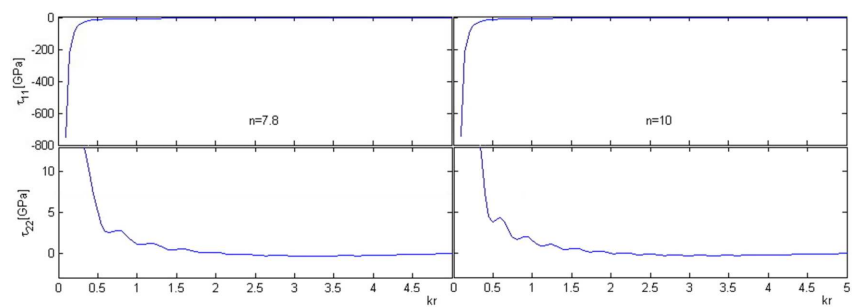

Fig. 2. Modelling of laser interaction. Numerical calculations of provoked stresses in semispherical geometry. Stress components are presented as a function of normalized distance $k \cdot r$, where $k$ is wave number.

self-focusing processes etc. could be treated separately. The real process evolution is often very complex, especially in the case of high power laser-material interaction.

The most frequently applied numerical calculations are thermal models where the temperatures of melting, boiling and other phase transformations determine the boundary conditions. By approaching to any of the critical points, i.e. damage threshold or disintegration energy, we have to take nonlinear effects into account. Many issues include the comparison between old phenomenological formulae derived from mechanics and microphysics [11] and the appropriate parts of macroscopic fracture 
theories. The calculation of values for provoked stresses has been performed by using the model explained in $[9,10]$ and the results are presented in Fig. 2. It is based on the data for Q-switch lasers assuming the pulse shape to be cosine (unlike the approximations made by other authors - to be Dirac $\delta$, triangle or compound function). The diffraction limit for laser intensity and the focusing lens parameter are included in the modeling of the normalized stress evaluation by using the parameter $n=2 \pi a / \lambda_{1} f k$, where $a$ and $f$ are diaphragm radius and focal length of the focusing lens, $\lambda_{1}$ is the wavelength of the laser, $k$ is the wave number [9]. It is based on the continuous mechanical model of thermo-elastic waves and on the general thermal equations.

\section{Use of SPICE program for laser-matter interaction}

Starting from one-dimensional bio-heat transport equation

$$
\begin{aligned}
& \rho c_{\mathrm{t}} \frac{\partial T}{\partial t}=\frac{\partial}{\partial x}\left(k \frac{\partial T}{\partial x}\right)-c_{k} \omega_{m}(T) \rho_{k}\left(T-T_{a}\right)+Q_{m} \\
& \quad+P(x, t),
\end{aligned}
$$

where $\rho$ is density, $c_{\mathrm{t}}$ and $c_{k}$ are specific heat of tissue and blood respectively, $k$ - thermal conductivity, $\omega$ - perfusion coefficient, $Q_{m}$ and $P(x, t)$ are the energies from internal and external heat sources and $T$ is the temperature [12]. Analogous equation is obtained for the equivalent electrical circuit (Fig. 3a), if the temperature is replaced by the voltage. Thus, the heat transport equation can be solved by using the program packages for analysis of electrical circuits (like SPICE), due to the mathematical analogy between the physical (thermal) and electrical models [13]. The parameters of the equivalent circuit are calculated from the parameters of the biomaterials-tissue:

$$
c \rightarrow \rho c_{\mathrm{t}}, r \rightarrow \frac{1}{k}, i \rightarrow Q_{m}+P(x, t), g \rightarrow C_{k} \rho_{k} \omega_{m}
$$

In Fig. 3b, the characteristic time dependence of the temperature of epidermis is presented. In the modeling process common thermal constants are included. Let us note that by neglecting perfusion parameters the equation and electric circuits could be simpler and the results could be directly applicable in general material laser interaction modeling. Depending on the problem that is modeled various number of layers can be implemented.

\section{Inconsistencies, discussion and conclusion}

In [14], semiconductor treatments with two types of regime dynamics with varying polarization were reported. The morphology of the damages depended strongly on the polarization. In the experiments performed in vacuum with pure metals, composites and dielectrics, characteristic damage morphology was obtained, with a material collected at the walls of cells, used for the maintenance of low pressure. There is a

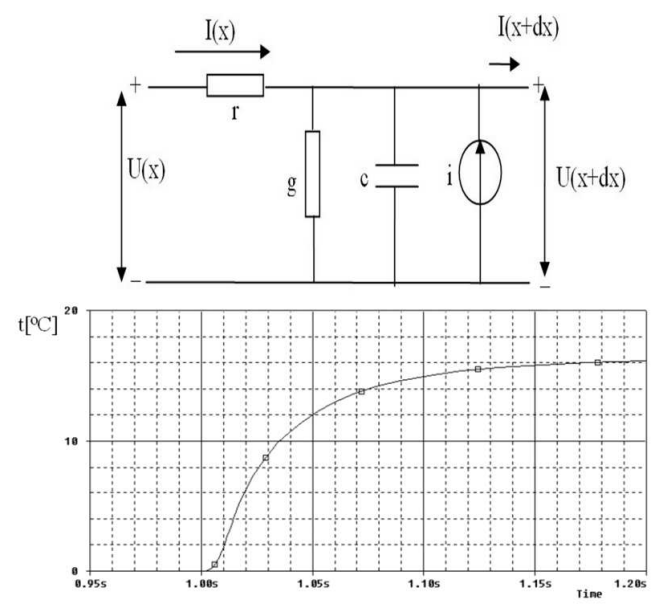

Fig. 3. (a) Equivalent circuit for three-layer epidermis; (b) time dependence of the temperature of epidermis for $x=40 \mu \mathrm{m}$.

huge amount of experimental data concerning the desorption [15] and obtaining of ultrafine powders with different regimes. Mentioned processes are described by many independent models. They can be applied in modern laser type-material interaction provided that the macroscopic constants for atomic, molecular parts used in some models are corrected.

In the analysis of hyperfast phenomena, significant attention should be paid to electron processes. By correcting the coefficients of reflection and absorption $(R, A)$, and specific heats $(c)$, and by knowing the spatial and temporal shapes of laser intensity, general thermal equations are applicable. However, chemical photoprocesses (such as oxidation) require another phenomenological approach.

Damage threshold can be studied by the use of fitting as well as statistics. We fitted some breakdown thresholds according to the data from references and concluded that there is a lot of space for discussion about the chosen fitting curve (for methods with 4 parameter tuning) [16]. The same is true for the threshold values in [17], because the experimental values and fitting procedures are not unique. It seems that the importance of the statistical nature of provoked damages is not taken into account correctly. Some inconsistencies exist when considering the theoretical approaches to facts, the experiments (following regular administrative procedure or not), the estimation of damage formation and the statistics.

Besides desorption, the production of particles and ion sources should have the common basics. Really, it should be a long way to unite the experiment and theories from various points of view (considering ejected material and considering the changes on damaged place). On the other hand, because ultrafast processes cannot be monitored with experimental techniques properly, all recorded data has to be analysed for the reliable reconstruction of interaction processes. It is not only about the establishing of 
the damage morphology, but also about the contribution to the complex physics of surface phenomena.

\section{Acknowledgments}

This work was supported by the Ministry of Sciences of Republic of Serbia, under numbers: 141009 and 11014 TR.

\section{References}

[1] R.W. Boyd, Nonlinear Optics, Elsevier, Amsterdam 2008.

[2] M. Sreckovic J. Ilić, A. Kovačević, S. Pantelić, Z. Lationović, N. Borna, V. Cosović, Acta Phys. Pol. A 112, 935 (2007).

[3] T.S. Narasimhamurty, Photoelastic and Electo-optic Properties of Crystals, Mir, Moscow 1984.

[4] Y.V. Afanasiev, B.N. Chichkov, N.N. Demchenko, V.A. Isakov, I.N. Zavestovskaya, Europhysics Conference Abstracts 25A, 2021 (2001).

[5] R. Penttilä, H. Pantsar, P. Laakso, 11th NOLAMP Conf., http://www.nolampjoin2007.fi .

[6] A.E. Chmel, Mater. Sci. Eng. B 49, 175 (1997).

[7] Ž. Tomić, M. Srećković, V. Zarubica, S. Cuuk, B. Djokić, J. Ilić, S. Ostojić, M. Dukić, M. Sredojević, Proc. Infoteh Conf. CD E-VII 14, 2008, p. 668.
[8] M. Srecković, Ž. Tomić, D. Družijanić, S. Pantelić, Z. Latinović, Lj. Vulićević, M. Zivković, Proc. Infoteh Conf. E1-b-1, 2009, p. 38.

[9] L.I. Mirkin, Physical Basis of Material Processing by Laser, Izd. Mosk. Univ., Moscow 1975 (in Russian).

[10] M. Srećković, A. Kovačević, M. Davidović, M. Dinulović, M. Kutin, A. Milosavljević, B. Djokić, Proc. SPIG, 2006, p. 243.

[11] A.V. Ivanov, The Strength of Optical Materials, Mashinostroenie, Leningrad 1989 (in Russian).

[12] Z. Deng, J. Liu, Numer. Heat Transfer B 42, 563 (2002).

[13] M. Milić, Electrical Modeling of Physical Processes, Bit inženjering, Belgrade 2004 (memorial edition, in Serbian).

[14] M. Srećković, P.M. Nikolić, B. Vedlin, V. Šijački Žeravčić, A. Brvnar Rožaj, P. Osmokrović, S. Vujatović, Exp. Techn. Phys. 37, 173 (1989).

[15] E.F. Lazneva, Laser desorption, Len. Uni, Leningrad 1990 (in Russian).

[16] S. Ostojić, R. Šašić, J. Opt. Adv. Mater. 84, 1402 (2006).

[17] R.M. Wool, Laser Damage in Optical Materials, A. Hilger, Bristol 1986. 